\title{
On the Dirac Structure of Confinement
}

\author{
Adam P. Szczepaniak ${ }^{1}$ and Eric S. Swanson ${ }^{1,2}$ \\ ${ }^{1}$ Department of Physics, North Carolina State University, Raleigh, North Carolina 27695-8202 \\ 2 Thomas Jefferson National Laboratory, 12000 Jefferson Avenue, Newport News, VA 23606
}

\begin{abstract}
The Dirac structure of confinement is shown to be of timelike-vector nature in the heavy quark limit of QCD. This stands in contradiction with the phenomenological success of the Dirac scalar confining potential. A resolution is achieved through the demonstration that an effective scalar interaction is dynamically generated by nonperturbative mixing between ordinary and hybrid $Q \bar{Q}$ states. The resolution depends crucially on the collective nature of the gluonic degrees of freedom. This implies that dynamical gluonic effects are vital when attempting to incorporate fine structure in models of the $Q \bar{Q}$ interaction.
\end{abstract}

Typeset using REVTEX 


\section{INTRODUCTION}

Although it has been postulated for more than 30 years, the phenomenon of quark confinement remains an enigmatic feature of QCD. Quenched lattice gauge theory and heavy quark phenomenology indicate that the static $\left(m_{q}>>\Lambda_{Q C D}\right)$ long range potential should be linear with a slope of $b \approx 0.18 \mathrm{GeV}^{2}$. The order $1 / m_{q}^{2}$ quark-antiquark long range spindependent (SD) structure has also been studied. Comparison with spin splittings in the $J / \psi$ and $\Upsilon$ spectra [1] indicate that the spin-dependence can only arise from the nonrelativistic reduction of a scalar current quark-antiquark interaction. This picture is also supported by calculations of the long range spin-dependent effective potentials on the lattice [2]

Unfortunately, little analytical progress has been made on this problem. The framework for most investigations on this subject was provided by the Wilson loop approach of Eichten and Feinberg [3] who extended the analysis of the spin-independent potentials by Brown and Weisberger [⿴囗口 . The standard parameterization for the long range SD quark-antiquark interaction introduced in Ref. [3] is given by

$$
\begin{aligned}
V_{S D}(r)= & \left(\frac{\boldsymbol{\sigma}_{q} \cdot \mathbf{L}_{q}}{4 m_{q}^{2}}-\frac{\boldsymbol{\sigma}_{\bar{q}} \cdot \mathbf{L}_{\bar{q}}}{4 m_{\bar{q}}^{2}}\right)\left(\frac{1}{r} \frac{d \epsilon}{d r}+\frac{2}{r} \frac{d V_{1}}{d r}\right)+\left(\frac{\boldsymbol{\sigma}_{\bar{q}} \cdot \mathbf{L}_{q}}{2 m_{q} m_{\bar{q}}}-\frac{\boldsymbol{\sigma}_{q} \cdot \mathbf{L}_{\bar{q}}}{2 m_{q} m_{\bar{q}}}\right)\left(\frac{1}{r} \frac{d V_{2}}{d r}\right) \\
& +\frac{1}{12 m_{q} m_{\bar{q}}}\left(3 \boldsymbol{\sigma}_{q} \cdot \hat{\mathbf{r}} \boldsymbol{\sigma}_{\bar{q}} \cdot \hat{\mathbf{r}}-\boldsymbol{\sigma}_{q} \cdot \boldsymbol{\sigma}_{\bar{q}}\right) V_{3}(r)+\frac{1}{12 m_{q} m_{\bar{q}}} \boldsymbol{\sigma}_{q} \cdot \boldsymbol{\sigma}_{\bar{q}} V_{4}(r) .
\end{aligned}
$$

Here $\epsilon=\epsilon(r)$ is the static potential, $r=|\mathbf{r}|=\left|\mathbf{r}_{q}-\mathbf{r}_{\bar{q}}\right|$ is the $\bar{Q} Q$ separation and the $V_{i}=V_{i}(r)$ are determined by electric and magnetic field insertions on quark lines in the Wilson loop expectation value (explicit expressions are given in Section II.C). As shown by Gromes [5] covariance under Lorentz transformation leads to a constraint exists between the SD potentials,

$$
\epsilon(r)=V_{2}(r)-V_{1}(r)
$$

Other more or less fundamental relations were also derived [3, 6, 7].

In a model approach, $Q \bar{Q}$ interactions are typically derived from a nonrelativistic reduction of a relativistic current-current interaction. As far as long range potentials are 
concerned only time-like vector or scalar currents are relevant [8]. Performing a nonrelativistic reduction of a vector-vector interaction yields $V_{1}=0, V_{2}=\epsilon, V_{3}=\epsilon^{\prime} / r-\epsilon^{\prime \prime}$, and $V_{4}=2 \nabla^{2} \epsilon$. Alternatively, the reduction of a scalar interaction yields

$$
V_{1}=-\epsilon, \quad V_{2}=V_{3}=V_{4}=0 .
$$

It is the alternation in sign of the combination $V_{1}+V_{2}$ between vector and scalar currents which, through the analysis of the heavy quarkonia spectrum, enabled Schnitzer to identify the scalar interaction as the likely structure for confinement [9].

Calculations [10,11] of the SD potentials based on sophisticated models of the Wilson loop typically yield results which are in agreement with Eq. (3). In particular the "minimal area law" model for the Wilson loop, a simple extension of the strong coupling limit of lattice QCD, leads to a picture which is very close to the classical flux tube model of Buchmüller [12. He noted that if one assumes that the chromoelectric field is confined within a tube between the $\bar{Q} Q$ pair then the magnetic field generated by the flux tube vanishes in the individual quark rest frames and the only contribution to the fine structure comes from the kinematical Thomas precession. This results in a SD structure which is identical to that of Eq. (3).

A consistent picture of a QCD-generated effective scalar confinement interaction appears to be emerging. It is therefore disconcerting that attempts to build Hamiltonian-based models of QCD [13] seem to require vector confinement. For example, we have found 14, 15 that it is impossible to construct a stable BCS-like vacuum of QCD when scalar confinement is assumed. This is problematical if one wishes to dynamically generate constituent quark masses. Furthermore, attempts at modeling chiral pions will be hindered by the explicit lack of chiral symmetry in a scalar interaction. These observations appear to stand in contradiction to the well established scalar confinement hypothesis.

In the following we resolve this issue by first performing a Foldy-Wouthuysen reduction of the full Coulomb gauge Hamiltonian of QCD. This immediately establishes that the Dirac structure of confinement for heavy quarks is of a timelike-vector nature. It is commonly 
stated in the literature that vector confinement is ruled out by its spin-dependent structure. We wish to stress that one must be careful in this judgement. In particular, the spindependent interactions which are generated by QCD are more complicated than those given by the simple nonrelativistic reduction of an effective long range interaction. Indeed, we shall demonstrate that the scalar character of the spin splittings in heavy quarkonia is dynamically generated through effective interactions which crucially depend on the collective nature of the gluonic degrees of freedom.

\section{HEAVY QUARK EXPANSION OF $H_{Q C D}$}

Our starting point is the Coulomb gauge QCD Hamiltonian [16]

$$
H_{Q C D}=\int d \mathbf{x} \psi^{\dagger}(\mathbf{x})[-i \boldsymbol{\alpha} \cdot \nabla+\beta m] \psi(\mathbf{x})+H_{Y M}+V_{C}-g \int d \mathbf{x} \psi^{\dagger}(\mathbf{x}) \boldsymbol{\alpha} \cdot \mathbf{A}(\mathbf{x}) \psi(\mathbf{x})
$$

where

$$
\begin{gathered}
V_{C}=\frac{1}{2} g^{2} \int d \mathbf{x} d \mathbf{y} \mathcal{J}^{-1} \rho^{a}(\mathbf{x}) V^{a b}(\mathbf{x}, \mathbf{y} ; A) \mathcal{J} \rho^{b}(\mathbf{y}), \\
V_{a b}(\mathbf{x}, \mathbf{y} ; A)=\left\langle\mathbf{x}, a\left|(\nabla \cdot \mathcal{D})^{-1}\left(-\nabla^{2}\right)(\nabla \cdot \mathcal{D})^{-1}\right| \mathbf{y}, b\right\rangle,
\end{gathered}
$$

and

$$
H_{Y M}=\frac{1}{2} \int d \mathbf{x}\left[\mathcal{J}^{-1} \mathbf{\Pi}(\mathbf{x}) \mathcal{J} \mathbf{\Pi}(\mathbf{x})+\mathbf{B}^{2}(\mathbf{x})\right]
$$

The degrees of freedom are the transverse gluon field $\mathbf{A}=\mathbf{A}^{a} \mathrm{~T}^{a}$, its conjugate momentum $\Pi=\Pi^{a} \mathrm{~T}^{a}$, and the quark field in the Coulomb gauge. The Faddeev-Popov determinant is written as $\mathcal{J}=\operatorname{Det}[\nabla \cdot \mathcal{D}]$, with $\mathcal{D}^{a b}=\nabla \delta^{a b}-g f^{a b c} \mathbf{A}^{c}$ being the covariant derivative in the adjoint representation, and the magnetic field is given by $\mathbf{B}^{a}=\nabla \times \mathbf{A}^{a}+g f^{a b c} \mathbf{A}^{b} \times \mathbf{A}^{c}$. The static interaction $V_{C}$ is the nonabelian analog of the Coulomb potential. It involves the full QCD color charge density which has both quark and gluon components,

$$
\rho^{a}(\mathbf{x})=\psi^{\dagger}(\mathbf{x}) \mathrm{T}^{a} \psi(\mathbf{x})+f^{a b c} \mathbf{A}^{b}(\mathbf{x}) \cdot \boldsymbol{\Pi}^{c}(\mathbf{x}) .
$$


The most salient feature of the Coulomb gauge Hamiltonian is that all of the degrees of freedom are physical. This makes it especially useful for identifying the physical mechanisms which drive the spin splittings in heavy quarkonia.

\section{A. The Foldy-Wouthuysen Transformation}

We proceed by performing a Foldy-Wouthuysen transformation on the QCD Hamiltonian. This is done in complete analogy to the quantum mechanical case where an operator is constructed which removes the interactions between upper and lower components of the

quark wave function order by order in the inverse quark mass [17], except that the unitary transformation is now constructed in Fock space. The resulting Hamiltonian is given by

$$
\begin{aligned}
H_{Q C D} \rightarrow H_{F W} & =\int d \mathbf{x}\left(m_{q} h^{\dagger}(\mathbf{x}) h(\mathbf{x})-m_{\bar{q}} \chi^{\dagger}(\mathbf{x}) \chi(\mathbf{x})\right)+H_{Y M}+V_{C}+H_{1}+H_{2}+\ldots, \\
H_{1} & =\frac{1}{2 m_{q}} \int d \mathbf{x} h^{\dagger}(\mathbf{x})\left(\mathbf{D}^{2}-g \boldsymbol{\sigma} \cdot \mathbf{B}\right) h(\mathbf{x})-\left(h \rightarrow \chi ; m_{q} \rightarrow m_{\bar{q}}\right), \\
H_{2} & =\frac{1}{8 m_{q}^{2}} \int d \mathbf{x} h^{\dagger}(\mathbf{x}) g \boldsymbol{\sigma} \cdot[\mathbf{E}, \times \mathbf{D}] h(\mathbf{x})+\left(h \rightarrow \chi ; m_{q} \rightarrow m_{\bar{q}}\right) .
\end{aligned}
$$

In this expression $h=(1+\beta) \psi / 2$ and $\chi=(1-\beta) \psi / 2$ denote the upper and lower components of the quark wave function and correspond to the annihilation and creation operators of the heavy quark and antiquark respectively. The ellipsis denotes terms which are either of $O\left(1 / m^{3}\right)$ or are spin-independent at order $1 / m^{2}$. Finally $\mathbf{D}=i \nabla+g \mathbf{A}$ is the covariant derivative in the fundamental representation. The electric field contains both transverse and longitudinal components, $\mathbf{E}^{a}=-\boldsymbol{\Pi}^{a}+\mathbf{E}_{\|}^{a}$, where

$$
\mathbf{E}_{\|}^{a}=-\nabla A_{0}^{a}-g \nabla \nabla^{-2} f^{a b c} \mathbf{A}^{b} \cdot \nabla A_{0}^{c}
$$

and

$$
A_{0}^{a}(\mathbf{x})=g \int d \mathbf{y} V^{a b}(\mathbf{x}, \mathbf{y} ; A) \rho^{b}(\mathbf{y})
$$




\section{B. The Static Potential}

To leading order in the quark mass the Hamiltonian describes two static, noninteracting quarks. At $\mathcal{O}\left(m^{0}\right)$ the Hamiltonian reduces to $H_{0}=H_{Y M}+V_{C}$. The eigenstates of $H_{0}$ may be labeled by the quark and antiquark coordinates and by an index which classifies the adiabatic state of the gluonic degrees of freedom, $n_{r}$,

$$
H_{0}\left|n_{r} ; \mathbf{r}_{q} \mathbf{r}_{\bar{q}}\right\rangle=\epsilon_{n}(r)\left|n_{r} ; \mathbf{r}_{q} \mathbf{r}_{\bar{q}}\right\rangle
$$

Note that we have made explicit the dependence of the gluonic degrees of freedom on the position of the quarks, $r$.

The corresponding eigenenergies, $\epsilon_{n}(r)$ may be identified with the Wilson loop potentials calculated on the lattice. Thus for example, $\epsilon_{0}(r)$ is the Coulomb plus linear potential seen long ago [18]. Static hybrid states are collectively denoted $\left|n_{r} ; \mathbf{r}_{q} \mathbf{r}_{\bar{q}}\right\rangle$ with $n_{r} \neq 0$. In recent studies [19] the lowest lying adiabatic hybrid potential, $\epsilon_{1}(r)$ has been evaluated.

While both $H_{Y M}$ and $V_{C}$ may contribute to the linearly rising potential energy seen on the lattice, it is clear that the quarks may only interact with the flux tube via the nonabelian Coulomb interaction. Therefore the Dirac structure of confinement corresponds to $\gamma_{0} \otimes \gamma_{0}$ from the product of color charge densities (see Eqs. (5) and (8)). As stressed in the Introduction, this appears to be at odds with 20 years of quark model phenomenology. Since the phenomenology is based on spin splittings, it will be instructive to examine the $1 / m^{2}$ perturbative corrections to the static potential.

\section{Spin-dependent Potentials}

The spin-dependent first order correction to the static potential is given by

$$
\delta \epsilon_{n}^{(1)}(r)=\frac{g}{8 m_{q}^{2}}\left\langle n_{r} ; \mathbf{r}_{q} \mathbf{r}_{\bar{q}}\left|\int d \mathbf{x} h^{\dagger}(\mathbf{x}) \boldsymbol{\sigma} \cdot[\mathbf{E}, \times \mathbf{D}] h(\mathbf{x})\right| n_{r} ; \mathbf{r}_{q} \mathbf{r}_{\bar{q}}\right\rangle+\left(h \rightarrow \chi ; m_{q} \rightarrow m_{\bar{q}}\right)
$$

The order $1 / m$ term is not considered because $\mathbf{D}^{2}$ is not spin-dependent and the matrix element of $\boldsymbol{\sigma} \cdot \mathbf{B}$ vanishes. Eq. (15) may be simplified considerably as follows. Since we are 
interested in spin-dependent terms only, the covariant derivative may be replaced with the ordinary derivative, $i \nabla$, and the electric field may be replaced by $-\nabla A^{0}$. Contracting the fermion field operators and using Eq. (12) yields

$$
\delta \epsilon_{n}^{(1)}(r) \sim \frac{-i g^{2}}{8 m_{q}^{2}} \epsilon^{i j k} \sigma_{q}^{i}\left\langle n_{r}\left|\nabla_{r_{q}}^{j}\left(\mathrm{~T}^{a} V^{a b}\left(\mathbf{r}_{q}, \mathbf{r}_{\bar{q}} ; A\right) \mathrm{T}^{b}\right)\right| n_{r}\right\rangle \nabla_{r_{q}}^{k}+\left(h \rightarrow \chi ; m_{q} \rightarrow m_{\bar{q}} ; \sigma_{q} \rightarrow-\sigma_{\bar{q}}\right),
$$

where the matrix elements are over gluonic degrees of freedom only. The approximation sign is meant to serve as a reminder that the equality holds for spin dependent terms only. The above equation may be simplified further by using the following relation

$$
\left\langle n_{r}\left|\left(\nabla_{r_{q}}^{j} g^{2} \mathrm{~T}^{a} V^{a b}\left(\mathbf{r}_{q}, \mathbf{r}_{\bar{q}} ; A\right) \mathrm{T}^{b}\right)\right| n_{r}\right\rangle=-\nabla_{r_{q}}^{j} \epsilon_{n}(r)
$$

The physical content of this relationship is simply the statement that $H_{Y M}$ does not explicitly depend on the quark positions. The minus sign is due to contracting the antiquark operators. Quark line contraction also yields tadpole terms which vanish in the color singlet background and self energy diagrams which are subsumed into the leading order, $\mathcal{O}(m)$, Hamiltonian. Making the appropriate substitution yields the standard classical plus Thomas precession spin orbit interaction

$$
\delta \epsilon_{n}^{(1)}=\left(\frac{\boldsymbol{\sigma}_{q} \cdot \mathbf{L}_{q}}{4 m_{q}^{2}}-\frac{\boldsymbol{\sigma}_{\bar{q}} \cdot \mathbf{L}_{\bar{q}}}{4 m_{\bar{q}}^{2}}\right) \frac{1}{r} \frac{d \epsilon_{n}}{d r} .
$$

Thus the first term in Eq. (1), generalized to any adiabatic potential and therefore true for both ordinary and hybrid $\bar{Q} Q$ states, is reproduced. At second order in perturbation theory the SD corrections are given by

$$
\delta \epsilon_{n}^{(2)}(r)=\sum_{m \neq n} \frac{\left|\left\langle n_{r} ; \mathbf{r}_{q} \mathbf{r}_{\bar{q}}\left|H_{1}\right| m_{r} ; \mathbf{r}_{q} \mathbf{r}_{\bar{q}}\right\rangle\right|^{2}}{\epsilon_{n}(r)-\epsilon_{m}(r)} .
$$

In this expression there are two terms which correspond to the application of the magnetic field operator twice on a single quark or antiquark line. These matrix elements are not spin dependent since the product of the Pauli matrices collapses to unity plus a single Pauli matrix. Alternatively, the case where the magnetic fields act on different quark lines is nontrivial and yields 


$$
\begin{aligned}
& \left.\delta \epsilon_{n}^{(2)}\right|_{B B}=\frac{g^{2}}{4 m_{q} m_{\bar{q}}} \sigma_{q}^{i} \sigma_{\bar{q}}^{j} \times \\
& \left(\sum_{m \neq n} \frac{\left\langle n_{r} ; \mathbf{r}_{q} \mathbf{r}_{\bar{q}}\left|\int d \mathbf{x} h^{\dagger}(\mathbf{x}) B^{i}(\mathbf{x}) h(\mathbf{x})\right| m_{r} ; \mathbf{r}_{q} \mathbf{r}_{\bar{q}}\right\rangle\left\langle m_{r} ; \mathbf{r}_{q} \mathbf{r}_{\bar{q}}\left|\int d \mathbf{y} \chi^{\dagger}(\mathbf{y}) B^{j}(\mathbf{y}) \chi(\mathbf{y})\right| n_{r} ; \mathbf{r}_{q} \mathbf{r}_{\bar{q}}\right\rangle}{\epsilon_{n}(r)-\epsilon_{m}(r)}+\right. \\
& \quad(h \leftrightarrow \chi)) .
\end{aligned}
$$

If we define $\left(g^{2}\right.$ times) the term in brackets as $\left[\left(\hat{r}^{i} \hat{r}^{j}-\frac{1}{3} \delta^{i j}\right) V_{3}+\frac{1}{3} \delta^{i j} V_{4}\right]$ then the third and fourth terms in the expression of the spin-dependent potential in Eq. (间) follow. If time-dependent fields are considered Eq. (20) may be rewritten as follows

$$
\left[\left(\hat{r}^{i} \hat{r}^{j}-\frac{1}{3} \delta^{i j}\right) V_{3}+\frac{1}{3} \delta^{i j} V_{4}\right]=\lim _{T \rightarrow \infty} g^{2} \int_{-T / 2}^{T / 2} d t d t^{\prime}\left(\frac{1}{T}\right)\left\langle n_{r} ; \mathbf{r}_{q} \mathbf{r}_{\bar{q}}\left|B^{i}\left(\mathbf{r}_{q}, t\right) B^{j}\left(\mathbf{r}_{\bar{q}}, t^{\prime}\right)\right| n_{r} ; \mathbf{r}_{q} \mathbf{r}_{\bar{q}}\right\rangle .
$$

Setting $n_{r}=0$ in this expression yields a result which agrees with that of Eichten and Feinberg叶.

There are four terms in Eq. (19) which contribute to $V_{1}$. These involve the application of the magnetic field and the covariant derivative on the same quark line,

$$
\begin{aligned}
& \frac{-g}{4 m_{q}^{2}}\left(\sum_{m \neq n} \frac{\left\langle n_{r} ; \mathbf{r}_{q} \mathbf{r}_{\bar{q}}\left|\int d \mathbf{x} h^{\dagger}(\mathbf{x}) \mathbf{D}^{2} h(\mathbf{x})\right| m_{r} ; \mathbf{r}_{q} \mathbf{r}_{\bar{q}}\right\rangle\left\langle m_{r} ; \mathbf{r}_{q} \mathbf{r}_{\bar{q}} \mid \int d \mathbf{y} h^{\dagger}(\mathbf{y}) \boldsymbol{\sigma} \cdot \mathbf{B}(\mathbf{y}) h(\mathbf{y})_{\mid} n_{r} ; \mathbf{r}_{q} \mathbf{r}_{\bar{q}}\right\rangle}{\epsilon_{n}(r)-\epsilon_{m}(r)}+\right. \\
& \text { H.c. } \left.+\left(h \rightarrow \chi ; m_{q} \rightarrow m_{\bar{q}}\right)\right) .
\end{aligned}
$$

Using the following relationship (which holds for the spin dependent terms only)

$$
\frac{d}{d t} h^{\dagger}(\mathbf{x}) \mathbf{D}^{2} h(\mathbf{x}) \sim-2 i g h^{\dagger}(\mathbf{x}) \mathbf{E}(\mathbf{x}) \cdot \nabla h(\mathbf{x})
$$

allows one to replace the first factor in Eq. (22) with one involving the electric field. Performing the time integral and contracting the fermion field operators results in $\left.\delta \epsilon_{n}^{(2)}\right|_{V_{1}}=\frac{g^{2}}{2 m_{q}^{2}} \sigma_{q}^{j}\left(\sum_{m \neq n} \frac{\left\langle n_{r}\left|E^{i}\left(\mathbf{r}_{q}\right)\right| m_{r}\right\rangle\left\langle m_{r}\left|B^{j}\left(\mathbf{r}_{q}\right)\right| n_{r}\right\rangle}{\left(\epsilon_{n}(r)-\epsilon_{m}(r)\right)^{2}}+H . c.\right) \nabla_{r_{q}}^{i}+\left(h \rightarrow \chi ; m_{q} \rightarrow m_{\bar{q}} ; \sigma_{q} \rightarrow-\sigma_{\bar{q}}\right)$.

\footnotetext{
${ }^{1}$ Their expressions are in terms of Wilson loops and therefore project onto the ground state as $T$ goes to infinity
} 
If $g^{2}$ times the term in brackets is defined as

$$
-i \frac{d V_{1}^{(n)}(r)}{d r} \frac{r^{k}}{r} \epsilon^{i j k}
$$

then this yields a spin orbit interaction in agreement with the second term in Eq. (1D). Alternatively, if the time integral is retained after substituting Eq. (23) in Eq. (22) and the sum over energy denominators is also represented by another time integral, then the expression for $V_{1}$ can be cast into the following form

$$
\hat{\mathbf{r}} \frac{d V_{1}}{d r}=\lim _{T \rightarrow \infty} \frac{i g^{2}}{2} \int_{-T / 2}^{T / 2} d t d t^{\prime}\left(\frac{t^{\prime}-t}{T}\right)\left\langle n_{r} ; \mathbf{r}_{q} \mathbf{r}_{\bar{q}}\left|\mathbf{B}\left(\mathbf{r}_{q}, t\right) \times \mathbf{E}\left(\mathbf{r}_{q}, t^{\prime}\right)\right| n_{r} ; \mathbf{r}_{q} \mathbf{r}_{\bar{q}}\right\rangle
$$

which agrees with the result of Eichten and Feinberg [3].

The remaining contributions to the second order energy correction correspond to an interaction between the quark and antiquark with both electric and magnetic operators at the vertices. Similar manipulations as for $V_{1}$ yield the portion of $V_{S D}$ proportional to $V_{2}$ once the following definition is made:

$$
-i \frac{d V_{2}^{(n)}(r)}{d r} \frac{r^{k}}{r} \epsilon^{i j k}=g^{2}\left(\sum_{m} \frac{\left\langle n_{r}\left|B^{i}\left(\mathbf{r}_{q}\right)\right| m_{r}\right\rangle\left\langle m_{r}\left|E^{j}\left(\mathbf{r}_{\bar{q}}\right)\right| n_{r}\right\rangle}{\left(\epsilon_{n}(r)-\epsilon_{m}(r)\right)^{2}}+H . c .\right),
$$

which is equivalent to

$$
\hat{\mathbf{r}} \frac{d V_{2}}{d r}=\lim _{T \rightarrow \infty} \frac{i g^{2}}{2} \int_{-T / 2}^{T / 2} d t d t^{\prime}\left(\frac{t^{\prime}-t}{T}\right)\left\langle n_{r} ; \mathbf{r}_{q} \mathbf{r}_{\bar{q}}\left|\mathbf{B}\left(\mathbf{r}_{\bar{q}}, t\right) \times \mathbf{E}\left(\mathbf{r}_{q}, t^{\prime}\right)\right| n_{r} ; \mathbf{r}_{q} \mathbf{r}_{\bar{q}}\right\rangle .
$$

The Faddeev-Popov determinants have not been carried through the calculations shown above. They may easily be restored without changing any of the results. The time integral representations of the potentials shall prove convenient for subsequent calculations; however they are rather opaque. Alternatively, the application of the Foldy-Wouthuysen transformation to the Coulomb gauge QCD Hamiltonian shows that these may be simply interpreted as nonperturbative mixing with hybrid states. This makes it clear that it is possible for nonperturbative dynamical physics to generate an effective spin-dependent interaction which mimics a scalar interaction. Actually demonstrating this requires that the matrix elements be evaluated. In the next section we propose to do this with the Flux Tube Model of Isgur and Paton. 


\section{MODEL EVALUATION OF THE SPIN-DEPENDENT POTENTIALS}

Before proceeding to a model evaluation of the matrix elements we note that it is possible to make some general statements on their expected properties. The results of lattice gauge theory make it clear that a flux tube-like configuration of glue exists between static quarks. If one thinks of this as a localized object with an infinite number of degrees of freedom, then it is apparent that $V_{2}$ must evaluate to zero. This is because the electric field operator creates a local excitation in the flux tube at position $\mathbf{r}_{q}$ (cf., Eq. (28)). This must then be de-excited at $\mathbf{r}_{\bar{q}}$ by the magnetic field operator. However, the two operators become decorrelated because infinitely many degrees of freedom intervene. Similarly, the long range portions of $V_{3}$ and $V_{4}$ both vanish. Thus, by Gromes' relation (Eq. (2)), the only nonzero long range interaction must be given by $V_{1}=-\epsilon$. This is precisely the situation required for "scalar" confinement. It is therefore entirely plausible that an effective scalar confinement is generated by nonperturbative mixing with hybrids. Furthermore, the structure of the spin-dependent terms depends crucially on the nature of the ground state gluonic degrees of freedom and clearly favors a collective rather than a single particle picture of them.

These simple expectations are borne out in an explicit model calculation. We shall employ the Flux Tube Model of Isgur and Paton [20] for this purpose. The model is extracted from the strong coupling limit of the QCD lattice Hamiltonian. The authors first split the Hamiltonian into blocks of distinct "topologies" (in reference to the possible gauge invariant flux tube configurations) and then make adiabatic and small oscillation approximations of the flux tube dynamics to arrive at an N-body discrete string-like model Hamiltonian for gluonic degrees of freedom. This is meant to be operative at intermediate scales $a \sim b^{-1 / 2}$ where the strong coupling is order unity. The lattice spacing is denoted by $a$ and there are $N$ "beads" (or links) evenly spaced between the $Q \bar{Q}$ pair. These considerations led Isgur and Paton to write the following model Hamiltonian

$$
H_{F T}=b_{0} r+\sum_{n=1}^{N} \frac{1}{2 b_{0} a} \mathbf{p}_{n}^{2}+\frac{b_{0} a}{2} \sum_{n=1}^{N+1}(\boldsymbol{\chi}(n)-\boldsymbol{\chi}(n-1))^{2}
$$


Here $b_{0}$ is the bare string tension, $\mathbf{p}_{n}$ is the conjugate momentum, and $\chi(n)$ is the transverse displacement vector at site $n$. It has been generalized to include color degrees of freedom: $\chi=\chi_{\lambda}^{c}(n), c=1 \cdots 8, n=1 \cdots N$, and $\lambda=1,2$. The ends of the string are fixed at $n=0$ and $n=N+1$ by the static quark and antiquark positions and $r=a(N+1)$.

In normal coordinates the Flux Tube Hamiltonian is

$$
H_{F T}=b_{0} r+\sum_{n=1}^{N} \sum_{\lambda=1}^{2}\left(\frac{1}{2 b_{0} a} \pi_{n \lambda}^{2}+b_{0} a \omega_{n}^{2} q_{n \lambda}^{2}\right)
$$

where the normal mode frequencies are given by $\omega_{n}=\frac{2}{a} \sin \left(\frac{n \pi}{2(N+1)}\right)$. This Hamiltonian may be trivially diagonalized with the following canonical transformation:

$$
\alpha_{n \lambda}^{a}=\sqrt{\frac{b_{0} a \omega_{n}}{2}} q_{n \lambda}^{a}+\frac{i \pi_{n \lambda}^{a}}{\sqrt{2 b_{0} a \omega_{n}}},
$$

and one obtains

$$
H_{F T}=\sum_{n \lambda} \omega_{n} \alpha_{n \lambda}^{\dagger} \alpha_{n \lambda}+b r-\frac{1}{a}-\frac{\pi}{12 r}+\ldots
$$

The string tension has been renormalized by the zero point energy which also introduces a (divergent) constant and terms higher order in $1 / r$ as shown above.

Evaluating the spin dependent potentials in the Flux Tube Model requires explicit expressions for the electric and magnetic fields in a flux tube. It is therefore necessary to extend the Flux Tube Model somewhat. We shall find it convenient to work in an "intermediate" coupling regime where we shall use the strong coupling lattice Hamiltonian for intuition and the weak coupling limit for the identification of the electric and magnetic fields. In the strong coupling limit the electric field operator simply counts links. It is therefore natural to map it onto a link displacement,

$$
E_{\lambda}^{a}(n) \sim \frac{\kappa}{a^{3}}\left(\chi_{\lambda}^{a}(n+1)-\chi_{\lambda}^{a}(n)\right)
$$

For the sake of clarity, we shall take $\mathbf{r}_{q}=\mathbf{0}$ and $\mathbf{r}_{\bar{q}}=r \hat{\mathbf{z}}$ from now on. This implies that $\lambda=x, y$. The factor $\kappa$ is an arbitrary constant and will be identified later.

The commutation relation between the electric and magnetic fields is 


$$
\left[E_{i}^{a}(\mathbf{x}), B_{j}^{b}(\mathbf{y})\right]=i \epsilon^{i j k} \nabla_{y}^{k} \delta(\mathbf{x}-\mathbf{y}) \delta^{a b}+\mathcal{O}(g)
$$

which implies that the magnetic field operator may be defined as the momentum conjugate to $\chi_{\lambda}$ [21]

$$
B_{\lambda}^{a}(n)=\frac{-i}{\kappa a} \frac{\partial}{\partial \chi_{\bar{\lambda}}^{a}(n)} .
$$

The $\epsilon^{i j k}$ of the commutator in Eq. (34) is taken into account by the index $\bar{\lambda}, \chi_{\bar{x}}(n)=-\chi_{y}(n)$ and $\chi_{\bar{y}}(n)=\chi_{x}(n)$. Note that this relationship is physically sensible because the magnetic field operator maps onto the plaquette in the strong coupling limit and the application of a plaquette to a flux tube has the effect of moving a link one unit in the one of the directions transverse to the $\bar{Q} Q$ axis with the magnetic field pointing in the other transverse direction.

Substituting Eqs. (33) and (35) into Eq. (29) and setting $\kappa=a \sqrt{b_{0}}$ yields

$$
H_{F T}=b_{0} r+\frac{1}{2} \int d \mathbf{x}\left(\left(E_{\perp}^{a}(\mathbf{x})\right)^{2}+\left(B_{\perp}^{a}(\mathbf{x})\right)^{2}\right) .
$$

This is reminiscent of the Coulomb gauge Hamiltonian where $H_{Y M}$ involves transverse fields only and where the nonabelian Coulomb interaction has been replaced with a linear potential. Thus a satisfying consistency has been achieved with this approach.

It is now a simple matter to write the field operators in terms of the collective phonon operators, $\alpha$. One obtains

$E_{\lambda}^{a}\left(\mathbf{x}_{n}, t\right)=\frac{\kappa}{a^{3} \sqrt{b_{0} r}} \sum_{m}\left(\sin \left(\frac{m \pi}{N+1}(n+1)\right)-\sin \left(\frac{m \pi}{N+1} n\right)\right) \frac{1}{\sqrt{\omega_{m}}}\left(\alpha_{m \lambda}^{a} \mathrm{e}^{-i \omega_{m} t}+\alpha_{m \lambda}^{a \dagger} \mathrm{e}^{+i \omega_{m} t}\right)$

and

$$
B_{\lambda}^{a}\left(\mathbf{x}_{n}, t\right)=\frac{-i}{\kappa} \sqrt{\frac{b_{0}}{r}} \sum_{m} \sin \left(\frac{m \pi}{N+1} n\right) \sqrt{\omega_{m}}\left(\alpha_{m \lambda}^{a} \mathrm{e}^{-i \omega_{m} t}-\alpha_{m \lambda}^{a \dagger} \mathrm{e}^{+i \omega_{m} t}\right)
$$

Substituting these expressions into Eqs. (26) and (28), taking the phonon operator matrix elements in the 0 -phonon number $\bar{Q} Q$ ground state, doing the time integrals, and evaluating the sum over modes yields 


$$
V_{1}(r)=-\frac{g^{2}}{2 a^{2}} C_{F} r
$$

and

$$
V_{2}(r)=\lim _{N \rightarrow \infty} \frac{g^{2}}{2 a^{2}} C_{F} \frac{r}{N}
$$

In the strong coupling limit one has $b=g^{2} /\left(2 a^{2}\right) C_{F}$ so that the anticipated expression for $V_{1}$ emerges in a natural way. Furthermore, $V_{2}$ approaches zero like $1 / N$; this is also true for $V_{3}$ and $V_{4}$. The latter point is illustrated in Fig. 1 where the correlation of electric and magnetic fields versus separation along the flux tube is shown. As expected the fields become completely decorrelated as the number of intervening degrees of freedom becomes large. Notice that this implies that a constituent gluon model of hybrids would not have been able to produce an effective scalar interaction.

\section{FIGURES}

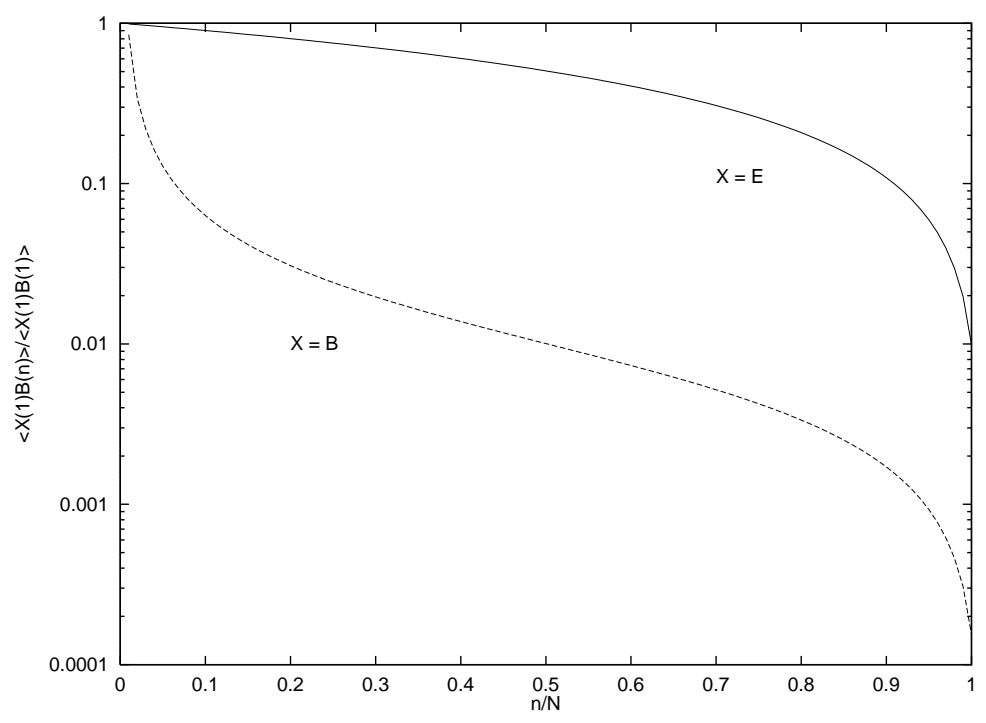

Fig. 1. Field Correlation Functions (for $\mathrm{N}=100$ ).

\section{CONCLUSIONS}

Spin splittings and lattice calculations indicate that confinement is scalar in nature. This conflicts with many relativistic models of QCD which require vector confinement. For example, a chirally symmetric interaction is needed if pseudo-Goldstone pions and spontaneous 
chiral symmetry breaking are to be generated dynamically. Furthermore it appears to be impossible to build a stable vacuum with a scalar kernel. We have examined this issue with the heavy quark limit of the Coulomb gauge QCD Hamiltonian. This approach is physically intuitive and is simpler to interpret and implement than methods based on the Wilson loop. We found that the static confinement potential must indeed be a Dirac timelike vector. Effective scalar interactions are generated at order $1 / m^{2}$ by nonperturbative mixing with hybrid states.

We have argued that the long range spin-spin $\left(V_{3}\right.$ and $\left.V_{4}\right)$ and the vector-like spin-orbit potentials $\left(V_{2}\right)$ should all be zero since they involve field correlation functions evaluated between quark and antiquark. This statement follows by assuming that the gluonic degrees of freedom collapse into a flux tube-like configuration, as shown by lattice gauge theory. Alternatively, the scalar-like spin-orbit potential $\left(V_{1}\right)$ is proportional to the matrix element of the electric and magnetic fields evaluated at the same point and hence is expected to be nonzero. Explicit calculations of the relevant matrix elements were carried out in the Flux Tube Model. The model was extended to include color degrees of freedom and to map the chromoelectric and chromomagnetic fields to flux tube phonon operators. The results obtained were in agreement with our general arguments and with Gromes' relation.

A consistent picture of the Dirac structure of confinement has emerged. The static central potential is timelike vector while the spin-dependent structure mimics the nonrelativistic reduction of an effective scalar interaction. This implies that it is incorrect to employ a scalar confinement kernel when doing calculations with light quarks. Note however that it would be acceptable to use scalar confinement when working explicitly in the chiral symmetry broken phase, i.e., with constituent quarks in the nonrelativistic limit. The work presented here also implies that a constituent gluon picture of hybrids will yield incorrect results for certain observables. For example, $V_{1}$ and $V_{2}$ would be of comparable magnitude in a constituent gluon model. In general, these types of models must fail when nonlocal properties of the gluonic configuration are considered. Alternatively, it is possible that they perform quite well when evaluating global properties of gluonics such as the hybrid spectrum. 
The application of these results to spin splittings in heavy quarkonia is not straightforward. For example, there is the possibility of large $\mathcal{O}\left(1 / \mathrm{m}^{3}\right)$ corrections to the splittings. Light quark loop effects may also contribute to spin-dependent forces. It is, unfortunately, rather difficult to quantify these effects. Perhaps the best hope is with high precision NRQCD lattice calculations. Light quark loops may be studied by examining shifts in the predicted spin splittings between quenched and unquenched calculations. One naively expects that these effects will be independent of the (heavy) valence quark mass. Alternatively, the higher order corrections should become larger as one moves from the $\Upsilon$ system to the $J / \psi$ system (the quenched approximation may used to test this). Lattice results for the quenched $\Upsilon$ spectrum [22] appear to be very close to experiment - supporting the idea that light quark loop effects are negligible (or at least may be absorbed into the parameters). Indeed, the calculated value of the ratio of P-wave splittings $\rho=\left[M\left({ }^{3} P_{2}\right)-M\left({ }^{3} P_{1}\right)\right] /\left[M\left({ }^{3} P_{1}\right)-M\left({ }^{3} P_{0}\right)\right]$ yields $0.7(3)$ [23], in good agreement with the experimental value of 0.66(2). Unfortunately, this may not be regarded as definitive since $\rho$ was evaluated at $\beta=6 / g^{2}=6.0$; a similar calculation at $\beta=5.7$ gives $\rho=1.4(4)$, very far from the experimental ratio. The situation in the $J / \psi$ spectrum is somewhat worse. There, Davies et al. [23] find $\rho=1.2(2)$, while the experimental value is $0.48(1)$. Thus it appears that higher order corrections in the inverse quark mass may become substantial at the charm quark scale. Clearly further lattice work is required to settle this issue. The effects of unquenching may also be studied theoretically (although this will necessarily be model dependent). For example, Eichten et al. [24] have used the Wigner-Weisskopf method in a vector confinement model to study the importance of virtual meson decays. Geiger and Isgur [25] have also studied such effects on the structure of the nucleon using the ${ }^{3} P_{0}$ model to incorporate meson loops.

The methodology which we have adopted here - using the heavy quark expansion of the Coulomb gauge QCD Hamiltonian to identify pertinent matrix elements and then using the (extended) Flux Tube Model to calculate these - is potentially very useful. For example, we may calculate the spin independent shifts to the static potential in precisely the same manner. This should prove interesting since we expect that they may not be obtained 
through the nonrelativistic reduction of an assumed kernel. Rather we will see for the first time evidence of the dynamical nature of confinement in the effective $1 / m^{2}$ structure. Spinorbit splittings in baryons have been something of a mystery for a long time [26]. These should be accessible with the same techniques used here - assuming that one can find a satisfactory quantum flux tube model of gluonic excitations in baryons. It should also be possible to examine the strong decays of heavy quarkonia with this approach. This is of interest since these processes are poorly understood and are of central importance to many phenomena of current interest. Finally, most current models of hadrons do not contain

dynamical (nonperturbative) gluonic degrees of freedom. It should, however, be possible to include them in a way which is consistent with the Flux Tube Model. We are currently investigating this possibility in the context of the Dynamical Quark Model [15].

\section{ACKNOWLEDGMENTS}

The authors are grateful to Nathan Isgur for fruitful discussions. ES acknowledges the financial support of the DOE under grant DE-FG02-96ER40944. 


\section{REFERENCES}

[1] H.J. Schnitzer, Phys. Rev. Lett. 35, 1540 (1975).

[2] C. Michael, Phys. Rev. Lett. 56, 1219 (1986).

[3] E. Eichten and F. Feinberg, Phys. Rev. D23, 2724 (1981).

[4] L. S. Brown and W. I. Weisberger, Phys. Rev. D20, 3239 (1979).

[5] D. Gromes, Z. Phys. C11, 147 (1981); erratum C14, 94 (1982).

[6] D. Gromes, Phys. Lett. B202, 262 (1988).

[7] C. Bachas, Phys. Rev. D33, 2723 (1986).

[8] W. Lucha, F. F. Schöberl, and D. Gromes, Phys. Rep. 200, 127 (1991).

[9] H. J. Schnitzer, Phys. Rev. Lett. 35, 1540 (1975).

[10] E. Montaldi, A. Burchielli, and G.M. Prosperi, Nucl. Phys. B296, 625 (1988); M. Baker, J.S. Ball, N. Brambilla, G.M. Prosperi, and F. Zachariasen, Phys. Rev. D54, 2829 (1996); Yu.A. Simonov, Nucl. Phys. B324, 67 (1989).

[11] N. Brambilla, P. Consoli, and G. M. Prosperi, Phys. Rev. D50, 5878 (1994).

[12] W. Buchmüller, Phys. Lett. 112B, 479 (1982). For extensions of this idea see M.G. Olsson, S. Veseli, and K. Williams, Phys. Rev. D53, 4006 (1996) and references therein.

[13] J.R. Finger and J.E. Mandula, Nucl. Phys. B199, 168 (1982); A. Le Yaouanc, L. Oliver, O. Pène, and J.-C. Raynal, Phys. Rev. D29, 1233 (1984); A. Le Yaouanc, L. Oliver, S. Ono, O. Pène, and J.-C. Raynal, Phys. Rev. D31, 137 (1985); S. Adler and P. Davis, Nucl. Phys. B244, 469 (1984); P.J. de A. Bicudo and J.E.F.T. Ribeiro, Phys. Rev. D42, 1611, 1625, 1635 (1990). For Schwinger-Dyson approaches see C.D. Roberts and A.G. Williams, Prog. Part. Nucl. Phys. 33, 477 (1994).

[14] A. Szczepaniak, E.S. Swanson, C.-R. Ji, and S.R. Cotanch, Phys. Rev. Lett. 76, 2011 
(1996).

[15] A.P. Szczepaniak and E.S. Swanson, From Current to Constituent Quarks: a Renormalization Group Improved Hamiltonian-based Description of Hadrons, hep-ph/9609525, to appear in Phys. Rev. D.

[16] T.D. Lee, Particle Physics and Introduction to Field Theory, (Harwood Academic Publishers, New York, 1981).

[17] F. Feinberg, Phys. Rev. D17, 2659 (1978).

[18] See e.g., the compilation in A. Hasenfratz and P. Hasenfratz, Ann. Rev. Nucl. Part. Sci., 35, 559 (1985).

[19] S. Perantonis and C. Michael, Nucl. Phys. B347, 854 (1990).

[20] N. Isgur and J. Paton, Phys. Rev. D31, 2910 (1985).

[21] A similar expression for the magnetic field is given in J. Merlin, D. Phil., Oxford University, 1986.

[22] C.T.H. Davies et al., Phys. Rev. D50, 6963 (1994).

[23] C.T.H. Davies et al., Phys. Rev. D52, 6519 (1995).

[24] E. Eichten, K. Gottfried, K.D. Lane, T. Kinoshita, and T.-M. Yan, Phys. Rev. D17, 3090 (1979); ibid 21, 203 (1980).

[25] P. Geiger and N. Isgur, Phys. Rev. D47, 5050 (1993); Strange Hadronic Loops of the Proton: a Quark Model Calculation, hep-ph/9610445.

[26] N. Isgur and G. Karl, Phys. Lett. 72B, 109 (1977). 